\title{
Chemical fingerprinting of biologically active compounds and morphological transformation during microwave reflux extraction of black pepper
}

Olusegun Abayomi Olalere a , Nour Hamid Abdurahman a , Rosli bin Mohd Yunus b, Oluwaseun Ruth Alara b , Nassereldeen Ahmed Kabbashi c

aCentre of Excellence for Advanced Research in Fluid Flow, University Malaysia Pahang, Pahang, Malaysia

bFaculty of Chemical Engineering and Natural Resources, Universiti Malaysia Pahang, Lebuhraya Tun Razak, Pahang 26300, Malaysia.

cInternational Islamic University Malaysia, KOE, BTE Engineering Department, Malaysia

\begin{abstract}
Emerging evidence has revealed the functional characteristics of black pepper extracts for both nutritional and medicinal purposes. The medicinal properties of this important commodity crop are due to the presence of active piperine which it a viable drug potential in ethnomedicine for the treatment of many degenerative free-radical related diseases. In this study, the microwave-enhanced technique was employed in the extraction of alkaloid oleoresins at optimum condition. The metabolomics-based approach was employed to determine the alkaloid profiles using LC-MS-Quadrupled time of flight mass spectrometer. At optimum microwave condition, a total of 21 bioactive alkaloids were identified with piperine being the most abundant with a mass to charge ratio of $286.1442 \mathrm{~m} / \mathrm{z}$. The result from the SEM-monograph and BET-surface characterization showed a remarkable transformation in the micro-cellular matrix as a result of the applied microwave electromagnetic heating.
\end{abstract}

